\title{
Module didactique basé sur l'Approche Par Compétences pour développer les compétences de la prise de notes en français chez les étudiants du diplôme général à la faculté de pédagogie
}

Recherche présentée par

Amani Ramadan Abdrabou ${ }^{1}$

\section{Résumé :}

La réception, l'assimilation et la mémorisation des cours ne sont pas chose aise pour la plupart. Nous avons pu découvrir qu'il existe des méthodes efficaces, pouvant faciliter cette activité de prise de notes pour l'intérêt de l'étudiant.

De même, offrir des outils pratiques et efficaces permettant d'améliorer ses habiletés en prise de notes de telle sorte que l'information et l'enseignement reçus soient plus efficaces. Il s'agit de savoir quoi faire "avant, pendant et après" la prise de notes.

Notre recherche se penche sur la méthodologie de la prise de notes, ses méthodes, ses techniques et ce à travers une unité que nous avons élaborée, relevant des problématiques essentielles rencontrées à leur niveau.

Nous avons essayé d'analyser les données que nous avons pu recueillir auprès des étudiants concernés, dans le but de démontrer l'intérêt et l'utilité de l'usage de la prise de notes tant sur le plan de l'assimilation, de la compréhension, mais aussi du processus de production (mettre en

\footnotetext{
${ }^{1}$ Maître de conférences de la didactique du FLE

Faculté de pédagogie

Université d'Ain Chams
} 
forme matériellement les informations jugées pertinentes, en les transcrivant rapidement), de confirmer l'occupation de la position essentielle dans l'apprentissage des savoirs, particulièrement à l'université, dans la mesure ou cette activité représente souvent un support de connaissances idéal pour préparer les examens. Les étudiants sont également convaincus de l'utilité primordiale de la prise de notes pour réussir leurs travaux.

D'ailleurs, plusieurs recherches (Ghovanlu, E. 2011 et Bouchet,K.2018) ont tenté d'explorer cette pratique afin d'en améliorer ses exploits. C'est-à-dire la prise de notes est personnelle et l'étudiant est autonome dans les techniques qu'ils utilisent pour cela et dans le choix des abréviations. L'essentiel est de garder des traces du cours auquel il assiste ou d'un ouvrage qu'il lit.

Mots clés : prise de notes, APC, FOU, FOS. 


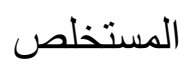

استهدفت الدراسة الحالية اقتر اح موديول قائم على مدخل الكفايات لتتمية بعض مهار ات

تدوين الملاحظات لدى طلاب الدبلوم العام بقسم اللغة الفرنسينة بكلية التربية جامعة عين شمس فئس ولتحقيق هذا الهدف أعدت الباحثة بطاقة تقويم لملاحظة وتحليل مهارات افراد العينة في هذا لتابه

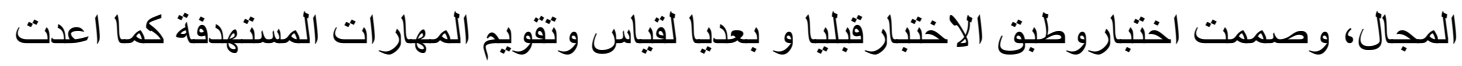
الباحثة موديول قائم على مدخل الكفايات لتتمية مهار ات تدوين الملاحظات. وقد أظهر التحليل

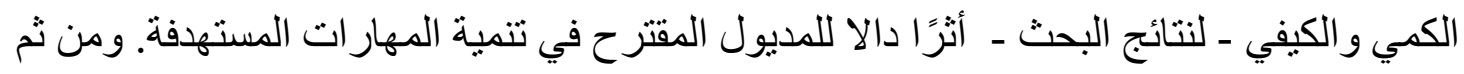

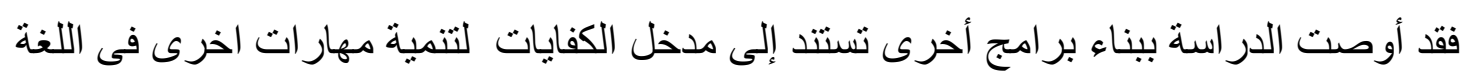
الفرنسية كلغة اجنيبة. 
Module didactique basé sur l'Approche Par Compétences pour développer les compétences de la prise de notes en français chez les étudiants du diplôme général à la faculté de pédagogie

Recherche présentée par

Amani Ramadan Abdrabou ${ }^{2}$

\section{Introduction:}

La langue française occupe une place cruciale dans le système éducatif égyptien. Le français général $\mathrm{FG}^{*}$ a pour objectif d'amener l'apprenant à communiquer dans différentes situations de la vie quotidienne. Il se caractérise par une formation à moyen ou à long terme avec une diversité de compétences à développer. L'enseignant de français général maîtrise les contenus de cours, travaille en autonomie et utilise des supports qui existent déjà.

À partir de 1960, on constate l'émergence d'un nouvel enseignement du français, qui est destiné à un public ayant des besoins particuliers. On a alors accorde un intérêt particulier aux domaines relatifs à la science et technique, au droit, a l'économie....etc. (Qotb,H.2013)

Le développement de ces enseignements particuliers a donné naissance à partir des années 80, mais surtout 90 au français sur objectifs spécifiques (FOS*), qui à son tour va donner naissance à plusieurs autres champs du français.(Hadriat H.2013)

* FG: Français général.

\footnotetext{
2 Maître de conférences de la didactique du FLE

Faculté de pédagogie

Université d'Ain Chams
} 
*FOS: Français sur objectifs spécifiques.

Le FOS vise à développer chez l'apprenant des compétences professionnelles. Il se caractérise par une formation à courte durée. L'enseignant ne maîtrise pas le contenu d'où la nécessité d'entrer en contact avec les acteurs professionnels, d'élaborer les matériels et les activités pédagogiques.

Effectivement, le FOS se diffère du FG au niveau lexical et des situations de communication, il demande une méthodologie différente par rapport à l'enseignement du FG et vise un public qui possède déjà des connaissances de base de la langue française.

Dans cette même dimension, nous avons un autre concept qui commence à gagner du terrain au domaine de la didactique des langues étrangères.

Il s'agit du FOU* français sur objectif universitaire. C'est une spécialisation au sein du FOS visant à préparer des étudiants à suivre des études supérieures dont la langue d'enseignement est le français.(Radwan,A.2017)

Le FOU, une branche du FOS, cherche à donner une nouvelle dimension au français et permanent aux étudiants d'acquérir des compétences langagières disciplinaires et méthodologiques. Le FOU concerne la vie à l'université, la compréhension orale du discours universitaire et l'expression orale.

Il concerne les étudiants qui veulent poursuivre leurs études dans des universités francophones ou bien dans leurs pays ou les études supérieures dans les branches scientifiques et techniques se font en 
*FOU: Français sur objectifs universitaires.

français, ce qui fait qu'ils ont besoin d'une formation en français de spécialité afin qu'ils puissent réussir dans leurs études.

D'où la nécessité de réfléchir à appliquer une approche qui permet à l'étudiant d'acquérir des compétences durables susceptibles de l'aider dans son parcours éducatif et met l'accent sur tout ce qu'est fondamental afin de garantir une meilleure transmission des savoirs. L'APC répond donc à tous les constituants de l'enseignement. Elle vise à mettre l'étudiant au centre du processus éducatif pour réussir ses apprentissages.

C'est une approche pédagogique active qui consiste en un apprentissage plus dynamique et plus durable. Elle met l'accent sur la compétence de l'apprenant d'utiliser concrètement ce qu'il a appris dans des situations nouvelles et complexes (Bruno,G.H. 2012; ElKhatib,W.2009). Elle consiste à définir les compétences dont chaque apprenant a besoin pour passer à l'étape suivante de son parcours universitaire, pour accéder à une qualification et pour être préparé à l'apprentissage tout au long de la vie.

L'APC utilise les compétences nécessaires dans un domaine comme point de départ dans la conception et le développement d'un curriculum ou une activité pédagogique.(Rogiers,X.2000)

Cette approche, fut adoptée dans le domaine de l'enseignement, est de plus en plus admise dans les systèmes éducatifs.Elle utilise des compétences nécessaires dans un domaine ou une pratique (l'enseignement universitaire) comme un point de départ dans la 
conception et le développement d'un curriculum, scénario ou activité pédagogique.

A ce propos, elle apporte une nouvelle vision à l'apprentissage et l'enseignement. Il s'agit de permettre aux étudiants de construire des compétences, c'est-à-dire de leur fournir un cadre lui permettant de développer des compétences (Scallon,G. 2004). Le bon étudiant dans l'APC sélectionne, fait des reformulations personnelles avec les savoirs et fait des synthèses.....

Les auteurs (Jacques, A. et Roxanne, R. 2003 ; Guillon, I.2000 et Etezadi G.2011) ajoutent que la prise de notes est comme un savoir-faire méthodologique qui aide l'étudiant à être autonome dans son travail et améliorer ses exploits pour réussir.

La prise de notes constitue une des difficultés majeures des étudiants d'études supérieures. Beaucoup ne sont pas capables de suivre des cours à l'université, faute de capacité à prendre des notes, ou éprouvent des difficultés à faire un exposé, élaborer une fiche de lecture, ou à produire un compte-rendu ou une synthèse de documents, etc. C'est à dire à recourir aux méthodes de travail indispensables au stade des études universitaires en général, et encore plus nécessaires dès lors qu'il s'agit de poursuivre ses études supérieures.

En effet, la prise de notes se trouve durant un apprentissage à la fois linguistique et méthodologique. Elle favorise la mémorisation et la compréhension de discours. Elle implique une étape de compréhension et conservation de l'information utile. Elle met en jeu des compétences discursives en relation avec des différents types de comportement que l'étudiant dont acquérir. 
De plus, les résultats des études antérieures (Janet, Ivonne 2018 ;Boch,F.1998; Girolami,R. 2008; et Nyee D.J. 2018) ainsi que notre observation comme enseignante au cours de méthodologie, nous alarment sur des lacunes fort importantes, citons quelques-unes:

- Les étudiants ne déterminent pas l'abréviation qui convient le mieux.

- Ils ne reformulent pas les paragraphes à leur propre style.

- Ils ne transforment pas le texte en schéma.

- Ils ne relisent pas les notes après le cours et les complètent.

- Ils ignorent les techniques de la prise de notes.

- Ils gardent rarement des traces du cours auquel ils assistent.

Toutes ces lacunes nous poussent à penser autrement du processus enseignement/apprentissage. L'idée de compétences a émergé lorsque l'on a voulu favoriser l'insertion sociale et professionnelle de l'étudiant, c'est-à-dire la volonté que les savoirs acquis en classe puissent être réutilisés dans les différentes situations de la vie quotidienne.. L'approche par compétences prépare l'apprenant à être un sujet actif et autonome, elle vise à l'amener à faire face à des situations d'imprévus en l'habituant à utiliser des connaissances préalables acquises. C'est dans ce but que 1'APC a été choisi : elle permet de développer une personnalité et des savoirs procéduraux ou des connaissances nouvelles.

Plusieurs recherches confirment que ces compétences de prise de notes sont acquiert par le biais des activités contextualisées où l'approche par compétences l'APC est au centre de l'apprentissage. Donc, il s'avère nécessaire d'expérimenter l'efficacité de l'APC sur les compétences de la prise de notes. 


\section{Problématique de la recherche:}

La problématique de la recherche actuelle réside dans la faiblesse des compétences de la prise de notes chez les étudiants du diplôme général au département du français à la faculté de pédagogie université Ain-Shams.

Ainsi, la présente recherche vise à répondre à la question principale suivante :

Comment élaborer un module didactique basé sur l'APC pour développer les compétences de la prise de notes chez les étudiants du diplôme général?

La réponse de cette question principale passe par une série de questions secondaires:

1. Jusqu'à quel point les étudiants du diplôme général se dotent des compétences de la prise de notes?

2. Quels sont les critères selon lesquels l'enseignant peut évaluer ces compétences?

3. Quels sont les caractéristiques d'un module à la lueur de l'APC pour développer les compétences visées chez ces étudiants?

4. Quelle est l'efficacité du module proposé sur le développement des compétences visées chez les membres de l'échantillon?

\section{Echantillon de la recherche:}

Notre échantillon concerne les étudiants du diplôme général de la faculté de pédagogie université Ain-Chams à l'année universitaire 2018-2019. 
مجلة دراسات في المناهج وطرق التّريس، الترقيم الدولي (ISSN 2535-213X)/ العدد 245 (2019))

\section{Délimitations de la recherche:}

la recherche actuelle se limite au développement de certaines compétences de prise de notes écrites chez les étudiants du diplôme général au département de français pendant l'année universitaire 20182019.

\section{Outils et matériels de la recherche:}

- Pré/post test qui vise à détecter et évaluer le niveau des membres de l'échantillon aux compétences de la prise de notes.

- $\quad$ Grille d'évaluation qui a pour but d'observer et d'analyser les pratiques des membres de l'échantillon lors de la prise de notes.

- $\quad$ Module proposé basé sur l'APC pour développer les compétences de la prise de notes.

\section{Hypothèses de la recherche:}

1- Il existe une différence significative entre les moyennes des notes des étudiants au pré / post test, en faveur du post test en ce qui concerne quelques compétences de la prise de notes.

2- Le module basé sur l'approche par compétences à une efficacité positive sur développement des compétences des membres de l'échantillon en ce qui concerne la prise de notes.

\section{Procédures de la recherche:}

Pour répondre aux questions de la recherche on va procéder comme suit :

1. Consulter les référentiels et les études antérieures concernant l'objet d'étude. 
2. Elaborer et valider le pré/post test pour détecter le niveau des membres de l'échantillon aux compétences de prise de notes.

3. Elaborer et valider la grille d'évaluation des compétences de la prise de notes.

4. Elaborer et appliquer le module didactique basé sur l'APC pour développer les compétences visées chez les membres de l'échantillon de la recherche.

5. Réappliquer le pré/post test auprès de l'échantillon de la recherche afin de tester l'efficacité de l'unité proposée.

5. Analyser les données statistiquement et interpréter les résultats.

6. Emettre les recommandations et les suggestions à la lueur des résultats obtenus.

\section{Terminologie de la recherche:}

\section{(1) Prise de notes:}

Selon le dictionnaire, le terme "prendre des notes" se définit par le fait de "sélectionner puis organiser l'essentiel d'une information et de la présenter de façon claire pour pouvoir la réutiliser".

La prise de notes désigne la transcription écrite résumée du discours oral. Les notes seraient de brèves indications recueillies par écrit en écoutant et en étudiant le discours oral. Elles ont pour principale fonction de ramasser les informations distribuées lors d'un discours oral dont il conviendra de se souvenir. (Piolat, A. 2006).

\section{(2) $A P C$ :}


Pour De Ketele (2000), l'APC est une approche qui cherche à développer la possibilité par les étudiants de mobiliser un ensemble intégré de ressources pour résoudre un problème dans se telle situation".

Roegiers,X. (2004) révèle que l'APC est une approche qui renferme les savoirs, les savoir-faire et les savoir être qui doivent être réinvestis dans des situations empruntées à la vie réelle.

\section{(3) APC et prise de notes:}

L'APC consiste à perfectionner les compétences de prise de notes chez les étudiants de FLE en leur formant à mobiliser et à développer des savoirs méthodologiques, des capacités d'écriture et de communication. Ce qui leur permet de relier les savoirs à leurs pratiques universitaires. (Définition opérationnelle)

\section{Cadre théorique:}

De nombreux étudiants qui viennent des systèmes universitaires différents, désirent bénéficier d'un accompagnement d'intégration universitaire qui se présente sous des diverses formes. Cette intégration réside dans une formation linguistique correspondant aux besoins générés par des situations langagières exigeantes. De plus, elle réalise à l'aide d'une méthodologie qui a pour but de répondre de la façon la plus correcte possible aux besoins réels d'un public, ça ce qu'on appelle FOU (Mangiante,J. et Parpette,C. 2004).

Le FOU dépasse les objectifs du français général, son objectif général est le comment : Comment prendre des notes, comment rédiger, comment faire un exposé, comment apprendre en autonomie, etc. 
Il se définit comme la branche du FOS dont le public visé est caractérisé par la pluralité des spécialités, dont les contenus sont principalement de type formel et procédural et dont les objectifs se subdivisent suivant trois axes: la vie de l'université, la compréhension orale du discours universitaire et les exigences de l'expression écrite.

En outre c'est une spécialisation qui vise à préparer des études supérieures dont la langue d'enseignement/apprentissage est le français. Il est caractérisé par certaines spécificités pour garantir l'efficacité de toute formation dans ce domaine. Ces spécificités concernent cinq points principaux :(Francis, I.\&Ruet,M.2014 ;Dufour,S.\&Parpette,C.2017)

1. La diversité des disciplines universitaires: Le Fou se distingue par la diversité des disciplines universitaires visées par les étudiants (droit, chimie, médecine, enseignement/apprentissage).

2. Les besoins spécifiques: Les publics déjà cités apprennent le français pour agir dans les différents milieux universitaires, les étudiants cherchent à comprendre les cours, prendre des notes, lire des livres spécialisés, passer des examens, rédiger des thèses. D'où la nécessité d'analyser leurs besoins avant l'élaboration des cours.

3. Le temps limité consacré à l'apprentissage: Les étudiants ont déjà leurs engagements universitaires .

4. La rentabilité de l'apprentissage du FOU: Les étudiants ont des objectifs précis à atteindre durant leur formation. Par exemple, ils sont motivés à suivre ce type de cours pour réussir dans leurs études universitaires. 
5. La motivation des publics: Les publics de Fou ont des objectifs bien précis dont la rentabilité dont être immédiate, ils montrent une grande motivation durant leur apprentissage. Alors, durant la formation il doit y avoir une réciprocité entre rentabilité et motivation.

Suivant cette idée, le Fou embrasse un domaine plus large que celui du FOS, qui est son parent théorique et pédagogique mais qui lui adapte son enseignement aux objectifs spécifiques plutôt que généraux. Il met l'accent sur les besoins spécifiques de communication et les pratiques de groupes particuliers dans des contextes universitaires (Radwan,A.2017 et Prunet,A. 2018). L'enseignement de base qu'il dispense porte sur la compréhension des exigences cognitives sociales et linguistiques de disciplines universitaires spécifiques.

Les praticiens du FOU doivent donc dépasser la préparation proprement linguistique des apprenants étrangers désireux de poursuivre des études en français pour développer chez eux des compétences communicatives leur permettant de participer à des activités dans des contextes universitaires et culturels variés. Etant fortement apparenté au FOS, le FOU reflète un grand nombre de points forts et de points faibles de son prédécesseur.

D'une part, il est caractérisé par le même accent mis sur une forte recherche interdisciplinaire comme moyen d'éclairer les contraintes des contextes sociaux sur l'utilisation de la langue et les façons dont les apprenants peuvent en prendre de contrôle. Il a également suivi les mêmes engagements clairs envers l'analyse linguistique, la pertinence contextuelle et la réplication de la classe des événements de communication de communautés spécifiques (Parpette,C.2004). 
D'autre part, il a également hérité de quelques-unes des limites discutées du FOS, en particulier une tendance à ne travailler qu'avec des spécialistes du sujet, une vulnérabilité aux affirmations selon lesquelles il ignore les cultures des étudiants et une réticence à engager une critique des valeurs, des buts et des pratiques institutionnels( Martin,R.2010).

Il est à noter que la réussite des programmes du français sur objectifs spécifiques (FOS) et du français sur objectifs universitaires (FOU) est jugée sur la qualité de l'engagement des étudiants dans ces programmes destinés à les aider. Pour réussir, ces programmes ont besoin d'être conçus avec grand soin en tenant compte des besoins précis des étudiants. En raison de la nature spécifique du FOU, ses méthodes d'enseignement doivent être distinctes de celles utilisées en français sur objectifs généraux, puisque les FOS/FOU sont habituellement programmés pour des périodes très limitées dans le temps (Albuquerque,C.,2015).

Alors, le FOS est une formation de courte durée qui vise à développer chez l'apprenant des compétences de communication professionnelle. Tandis que le FOU cherche à donner une nouvelle dimension au français langue de communication spécifique et permet aux étudiants d'acquérir des compétences langagières, disciplinaires et méthodologiques. En général, les domaines du Fou concernent la vie de l'université.

Ce travail analyse les difficultés ressenties par les étudiants dans le cadre de leur parcours universitaire. Il propose les bases pour l'élaboration d'un programme de français sur objectifs universitaires visant à préparer d'étudiants à l'acquisition de compétences linguistiques, 
disciplinaires et méthodologiques de la vie universitaire à partir de l'analyse de leurs besoins spécifiques :( Hilgert,E.2009; Mangiante,JM\& Parpette,C.2011)

* La composante linguistique: Il s'agit de développer chez les étudiants les connaissances linguistiques nécessaires (lexique, grammaire ...) pour que les étudiants puissent suivre les cours dans leurs domaines visés: comprendre le cours, prise des notes, poser des questions.

* La composante méthodologique: Les cours de FOU devraient développer chez les apprenants certaines compétences méthodologique visant à les aider à réaliser des tâches universitaires: participer à un colloque, préparer une communication, prendre la parole devant un public spécialisé, rédiger un article, déterminer une problématique, synthétiser un document, élaborer un plan de recherche, rédiger un mémoire ou une thèse ...

* La composante disciplinaire: Il s'agit de familiariser les étudiants avec leur domaine de spécialité en utilisant la langue cible. Dans ce contexte, les cours de FOU sont marqués par un échange entre les apprenants et leur enseignant, $\mathrm{Ce}$ dernier a les connaissances linguistiques et méthodologiques tandis que les étudiants maîtrisent le savoir spécialisé du domaine visé.

La prise de notes se trouve en effet durant un apprentissage à la fois linguistique et méthodologique. Elle favorise la mémorisation et la compréhension de discours. Elle implique une étape de compréhension et conservation de l'information utile. 
De ce fait, les praticiens du FOU doivent dépasser la préparation linguistique des étudiants étrangers de poursuivre des études en français pour développer chez eux des compétences communicatives qui leur permettent de participer à des activités dans le contexte universitaire et culturel variés.

L'objectif est de créer chez les étudiants des habiletés discursives qui leur permettant d'intégrer différentes manières dont s'élabore et se transmet le savoir à l'université et de déceler les spécificités des discours qu'ils fréquentent. Le FOU consiste à explorer les pratiques langagières par lesquelles le savoir circule à l'université.

Les premiers jours à l'université représentent un très grand changement pour de nombreux étudiants, en ce qui concerne les méthodes d'apprentissage auxquelles ils étaient habitués. Les professeurs ne dictent plus les cours, il n'y a pas de manuels scolaires et il faut savoir distinguer l'essentiel du superflu lors des cours magistraux.

L'étude de l'activité de prise de notes connaît un intérêt récent en France avec les travaux de chercheurs comme (Piolat,A. et Boch,F.2004), lorsqu'ils s'interrogent sur les fonctions de cette pratique dans le domaine de l'apprentissage, au domaine des disciplines comme la psychologie, la linguistique et la didactique de langues.

En effet, en situation de cours, il ne suffit pas pour les noteurs, d'écouter simplement l'enseignant délivrer des connaissances. Alors, il doit comprendre les informations transmises et les stocker en mémoire à long terme dans la perspective d'un réinvestissement efficace et pertinent le moment venu. On comprend que cette pratique a un caractère 
tridimensionnel d'écoute, de sélection et de compréhension des informations.

Ces futurs enseignants doivent être capables de produire tant des discours didactiques que des discours d'analyse d'une problématique. Cela concerne la formation de discours sur objectifs universitaires. Ces compétences sont transdisciplinaires et non réductibles à l'étude d'une langue de spécialité.

Pour bien mener cette activité, l'étudiant mobilise les stratégies de reformulation à l'écrit en recourant à plusieurs phénomènes tels que les abréviations, les sigles ou les transformations au niveau syntaxique. Ces effets qui sont propres à chacun, s'inscrivent dans la description de profils de noteurs spécifiques.

Lors de cette activité, le noteur doit être capable de traiter, sélectionner puis organiser l'essentiel de l'information et la présenter de façon claire pour pouvoir la réutiliser. Noter n'est pas recopier mais comprendre et rédiger.

C'est représenter pour mémoriser, par des signes graphiques, un condensé sélectif d'informations orales. Contrairement à la sténographie qui vise à transcrire intégralement la parole au rythme d'une prononciation normale, la prise de notes s'appuie rarement sur des signes conventionnels mais fait souvent appel à des marques et des symboles, que le scripteur reste libre de choisir (; Bessonnat,D.1995; Tremblay,R. et Perrier,Y.2000).

La prise de notes est un moyen utilisé dans le but de ne pas charger sa mémoire: elle est une compétence qui consiste à retenir le maximum d'informations en un temps limité (Vigouroux, 2007). 
Elle permet d'accroître notre attention au contenu véritable du cours plutôt que nous laisser distraire par tout ce qui se passe autour de nous pendant le cours, elle nécessite une capacité d'observation (discours du professeur) de consigner les observations faites en classe que l'étudiant doit effectuer tout le long de son parcours .

En un mot, prendre des notes c'est une compétence qui consiste à tirer l'essentiel de ce qu'on lit (textes - articles), voit (graphique, image fixe) ou entend (cours, exposé, enregistrement audio, message téléphonique). Il s'agit de sélecter ce qu'on doit retenir et d'éliminer le reste. Elle est aussi un savoir méthodologique qui aide l'apprenant à acquérir l'autonomie de son travail (Monique, 2000).

Nous prenons appui sur la prise de notes pour mettre en évidence l'hétérogénéité des compétences nécessaires à ce savoir-faire.

Ces savoir-faire doivent permettre une telle condensation syntaxique et textuelle en termes de lexique didactique et lexique de spécialité, gestion de la modalisation, cohésion, articulation logique, spécialités de l'oral, spécialités de l'écrit, subjectivation, objectivation.

La prise de notes se révèle différente selon que l'étudiant se trouve face à un document écrit ou face à un discours oral.

À l'écrit: Chercher dans le texte des idées directrices, repérer les articulations logiques de la pensée, le plan de l'exposé.

À l'oral: Écouter avec attention pour saisir les connecteurs logiques ou temporels (d'abord, ensuite, premièrement ...), les mots-clefs souvent répétés, les idées directrices souvent portées par la voix et le geste, les formules de conclusion et de transition. 
En situation de discours oral, il est très difficile de revenir en arrière, l'étudiant doit prendre des notes au fur et à mesure en s'adaptant au flot d'informations transmises par l'enseignant. Inversement, lorsque de discours est écrit et quel que soit la forme, la sélection des informations à noter n'est pas soumise à la cadence de la parole mais peut être reprise ou modifiée à tout moment. (Boch,F.2000 ; Dionne,B.2004)

En cette guise, la prise de notes suppose que l'on note au fil de l'exposé oral de l'enseignant ou au fil de sa propre lecture. Ce qui aide l'étudiant à comprendre, formuler et réviser, elle met en jeu des compétences discursives en relation avec différents types de comportements que l'étudiant doit acquérir avant, pendant et après:

Avant la prise notes, l'étudiant doit préparer son matériel (stylos, feuilles de papier ...) lire sur le thème. Pendant, il doit rester attentif tout le temps, repérer son plan autour des articulateurs, distinguer l'essentiel, repérer les mots clés, condenser le sens par nominalisations (champs lexicaux, mots de la même famille ...), utiliser des schémas, des réseaux, des flèches, ... c'est donc une activité qui force l'attention. Après, il doit poser des questions pour compléter les notes, ou mettre ces notes en valeur et trouver des titres et sous titres appropriés. C'est en dernier lieu qu'on écrit, relit reformule pour préparer un exposé ou un rendu ou conserver des notes pour une révision ultérieure.

Une fois ses cours réécris, on passe au rangement: classeur, portevue ou autre; on est capable de réviser un cours quand tout est ordonné et quand les informations sont à portée de main.

La prise de notes est un moyen de représenter des informations de sa propre façon. Elle impose d'écouter, et de réviser. La mémoire visuelle 
et auditive a été sollicitée durant les cours. Ainsi, elle remplit plusieurs objectifs:

Objectifs cognitifs: peuvent aller à la simple saisie de l'information à l'évaluation de celle-ci : s'informer sommairement du contenu du cours, s'informer du détail du contenu du cours, comprendre le contenu du cours et se préparer à l'évaluation des apprentissages.

Objectifs effectifs: Sont relatifs aux émotions qui précèdent, accompagnent et suivent la prise de notes tels que: se sécuriser, faire sentir au professeur que son cours est intéressant et se donner bonne conscience par le sentiment du devoir accompli.

Objectifs psychomoteurs: tiennent compte des habiletés sensomotrices: faire quelque chose d'utile pour demeurer attentif, s'informer du détail du contenu du cours et refréner le besoin de parler durant le cours.

\section{Comment réussir sa prise de notes?}

La prise de notes s'effectue en plusieurs temps: écouter comprendre, synthétiser et noter. Il faut donc stocker l'information, de ce fait, il sera peut-être utile d'améliorer son mémoire pour être plus efficace:(Bouchard,R.et Parpette,C.2007 ; Romainville,M.2000)

- S'entraîner à mémoriser des informations simples: données chiffrées, conserver en tête plusieurs numéros de téléphone, une liste de course, des noms propres ... etc.

- $\quad$ Se fixer des objectifs.

- Développer sa concentration et ses capacités d'observation.

- $\quad$ Utiliser des méthodes mnémotechniques ou de visualisation. 
Pour que la prise de notes soit lisible et claire, afin de ne pas perdre de temps à relire les notes et à les comprendre, il faut:

- Aérer les notes .

- Utiliser les mêmes repères et aux mêmes endroits.

- Numéroter les pages.

- $\quad$ Dater la prise de note et/ou les pages.

- Indiquer l'objet de la prise de notes.

De plus, il faut relire rapidement ses notes le même jour pour en vérifier la compréhension: note dans la marge les points obscurs, rectifier les abréviations inventées, compléter les "blancs" pendant les quels ton attention a baissé, les compléter avec les informations que tu as encore en mémoire, enfin, il est utile de comparer ses notes avec le manuel de manière à les compléter ou les rectifier si on a besoin.

Prendre des notes efficacement exige être attentif pour savoir les détecter, anticiper, c'est-à-dire revoir le cours la veille, se poser des questions et répondre aux questions en utilisant une diversité de techniques.

\section{Différentes techniques de prise de notes :}

Les techniques de la prise de notes permettent à l'étudiant la mémorisation d'informations lors de l'écoute d'un cours. De même, la prise de notes par le recours à un plan permet d'avoir une vue d'ensemble sur le cours et d'en repérer les idées directrices de ce cours. Ainsi, chaque étudiant développe son propre technique de prise de notes (; 
Piolat,A.\&Boch,F.2004, Piolat,A.2006 ). La prise de notes prend des formes différentes selon l'objectif et la personnalité de chacun.

\section{Forme linéaire:}

C'est une méthode spontanée qui consiste à retranscrire le discours sous forme des phrases placées à la suite les unes des autres. L'étudiant note plus de mots que de sens. Ainsi, il effectue plus tard le travail d'analyse et de synthèse consistant à tirer les idées principales des idées secondaires.(François-Xavier AirRault 2004).

\section{Forme structurée:}

C'est la méthode la plus familière et la plus traditionnelle (Ch. Voirol,1999). Elle repose sur le même principe que la précédente, mais sa disposition "en arbre" met encore en relief la structure, elle possède en plus l'avantage de laisser des espaces qui permettent de compléter les notes lors d'une relecture.

C'est une méthode spécifique qui permet de prendre des notes claires. De plus, elle stimule la motivation, car elle oblige à une écoute active. Cette technique exige que la page soit découpée en zones et chaque zone soit réservée à un type d'informations.

En effet, le premier tiers à gauche de la page sert pour les ajouts et que les deux autres tiers sont utilisés pour la prise de notes durant le cours. Le quart inferieur est prévu pour inscrire d'éventuelles questions d'examen.

\section{Forme heuristique:}


Les méthodes précédentes ont l'avantage de noter dans l'ordre ou elles sont reçues les différentes informations. Mais, il existe d'autres beaucoup plus dynamiques et souvent plus personnelles, comme la forme heuristique.

Elle offre une vue d'ensemble d'un sujet ou d'une situation et permet d'utiliser les fonctions globalistes du cerveau. Cette technique consiste à placer le thème principal au centre et les idées secondaires autour de ce thème. ( Brigitte Chevalier,1992). Il est plus facile de construire notre propre organisation.

De plus, un tel schéma est toujours ouvert: y introduire des idées nouvelles. Cette forme permet de définir clairement l'idée principale, ce qui est important est place près du centre et ce qui l'est moins est place en périphérique.(Elisabeth Lamont-Hoffmann, Magali-Monnier et AnneSylvie-Diezi,2007).

Les techniques de prise de notes sont multiples et l'efficacité de cette pratique réside plutôt d'en adopter l'une ou l'autre selon la situation et selon la nature du discours à noter. C'est pourquoi la chercheuse tente à utiliser plusieurs techniques tels que: le style télégraphique, les abréviations ou la carte mentale conformément à chaque compétence à développer.

\section{L'approche par compétences (APC):}

L'APC a trouvé son existence à la seconde moitié du XXe siècle comme réaction paradoxale de l'échec des modalités classiques de formation (absence des objectifs, tests écrits enfin du programme), elle a réalisé un grand succès dans les différents pays du monde. 
Cette approche a vu le jour grâce au remplacement du savoir par le savoir-faire, au développement de la technologie nouvelle, à l'expérimentation, au développement de modalités d'évaluation des enseignants, à l'innovation du concept d'apprentissage, à la pédagogie par la maîtrise.......etc.

De plus en plus, on évoque la nécessité de réfléchir à une approche par compétences l'APC dans nos universités. Notamment, plusieurs auteurs ( Boutin, G. \& Julien, L. ;Beacco, J-C 2011 ; Moulet, L.2011 Said, M.2015 ; Gohsmann, L. \& Délignieres, M. 2016) voient dans cette approche une sujétion de fait de la formation universitaire aux besoins des programmes d'études. Il est vrai que la mission traditionnelle de l'université est souvent la transmission de savoirs académiques et scientifiques, la démarche par compétences est suspectée de veiller à la professionnalisation de leurs diplômes.

La genèse de cette approche se résume dans le fait de définir les compétences à maitriser, ainsi que les critères à partir desquels on pourrait faire le contrôle.

L'APC requiert avant tout de modifier le rapport au savoir. On se contente encore souvent d'enseignement magistral au cours duquel les étudiants engrangent un ensemble de connaissances. L'APC suggère de veiller à ce que les étudiants s'entraînent à la mobilisation et le développement d'un ensemble de savoirs méthodologiques, de capacités d'écriture et de communication.

Dans l'APC, l'étudiant fait part de sa compréhension et montre son savoir-faire en désignant et en développant les choses avec ses propres mots (Richard,M. et Bissonnette,S. 2001). 
Bouhadiba, F. (2004) a souligné que l'APC offre meilleures chances de créer des situations porteuses de sens, du simple fait qu'elle relie les savoirs à des pratiques universitaires. A cet égard, (Koebel, M. 2006; Perrenoud, PH. 2004) évoquent que c'est aussi un moyen pour permettre aux étudiants de trouver davantage de sens à leurs études, et de lutter contre l'échec.

Les tenants de l'APC placent l'étudiant au centre de l'apprentissage. Ils considèrent l'étudiant comme naturellement doué d'une capacité presque absolue de développer les compétences visées qui apparaissent dans le programme d'études. L'étudiant est responsable de son apprentissage et il lui appartient de construire lui-même ses propres connaissances.

Pour ce faire, le rôle de l'enseignant est modifié en facilitateur. Il incite ses étudiants à construire leurs connaissances, il laisse une bonne partie de ses tâches au groupe-classe qui sera invité à procéder à son organisation. (, G.\&Julien, L.2000 ; Delignieres, D.2009 et Boutin)

Dans ce fait, il appartient à l'étudiant de déterminer lui-même les objectifs qu'il poursuit et l'enseignant doit éviter le plus possible la transmission de connaissance et même dissimiler à l'apprenant qu'il est soumis à une évaluation. L'accent est mis sur le développement des compétences de l'apprenant.

Autrement dit, le rôle de l'apprenant s'étend pour à recueillir de nouvelles informations, apprendre de nouvelles façons d'apprendre, savoir utiliser de nouvelles technologies (internet) pour atteindre de nouveaux objectifs et pour avoir accès à de nouvelles informations et les partager. 
De la part de l'enseignant, il devient un facilitateur qui planifie, organise des activités, conseille, accompagne, encourage, soutient, fait des suggestions mais n'impose jamais, stimule la créativité, encourage le développement d'une pensée indépendante. Le facilitateur soutient l'apprenant, tient compte de ses possibilités, de ses points forts, de ses besoins et de ses sentiments.

L'APC considère la formation de l'enseignant comme un processus continu, elle crée un milieu permissif d'apprentissage/enseignement, elle répond aux besoins varies des étudiants et elle adopte le principe d'intégration entre la théorie et la pratique. Dans ce contexte, il aide les étudiants à:(Perrenoud,PH.2004 ; Besançon,M.2004 et Koebel, M.2006)

- dresser des grilles pour les compétences que les chercheurs pourraient en prendre comme source de déduire les objectifs.

- renforcer les tendances efficaces telles que la pédagogie par objectifs, l'autonomie et les méthodes actives.

- entrainer l'enseignant à planifier et organiser son acte de classe de façon qu'il soit accessible à la réalisation des objectifs.

- identifier les interrelations qui existent entre les composants du programme (objectifs, contenu, stratégies d'enseignement, outils d'évaluation)

- préciser le rôle et les responsabilités de l'enseignant.

- s'intéresser à développer les compétences académiques, professionnelles et personnelles de l'enseignant. 
En un mot, l'APC permet à l'étudiant d'être capable de réaliser les activités, la prise en compte de diverses sources d'apprentissage(enseignement-formation-expérience),de développer chez l'étudiant un esprit d'apprentissage permanent et non pas limiter cet apprentissage a un moment de formation.

Dans ce contexte (Perrenoud.2007) définit cinq principes essentiels pour enseigner selon l'APC: créer des situations didactiques porteuses de sens et d'apprentissages, développer une observation formative et une régulation interactive en situation en travaillant sur les objectifs, maîtriser les effets de relations inter subjectives sur la communication didactique et individualiser les parcours de formation dans le cycle d'apprentissage.

En guise de conclusion, on peut résumer que l'APC est une manière de penser et de mettre en œuvre l'enseignement/apprentissage, centrée sur l'acquisition des savoirs et savoir-faire. C'est une notion qui vise à construire l'enseignant sur la base de savoir-faire, évalués dans le cadre de la réalisation d'un ensemble de tâches complexes. L'enseignement devient alors apprentissage.

L'APC vise à perfectionner les compétences de l'étudiant et améliorer sa productivité. C'est une méthodologie ciblée dans la mesure où elle fixe un référentiel de compétences à atteindre vers la fin de la formation.

\section{Etude expérimentale :}

Notre recherche d'expérimentation se compose de trois parties : après avoir brièvement défini le FOU et la prise de notes, nous présentons les méthodes sélectionnées, puis, nous procèderons à l'analyse du cadre 
théorique qui fera l'objet de notre expérimentation, enfin nous étudierons l'efficacité de l'APC à travers une démarche scientifique. Il s'agit d'identifier les besoins de notre public cible, de réajuster les activités didactiques, de faire passer une grille d'évaluation et appliquer le module proposé.

\section{1) Elaboration de la grille d'évaluation:}

Notre grille a pour but d'observer et d'analyser les pratiques des étudiants du diplôme général au département du français à la faculté de pédagogie à l'université Ain-Chams lors de la prise de notes : de même, elle peut être utilisée comme un outil d'auto évaluation afin que les étudiants des études supérieures prennent conscience de leurs points forts et faibles en ce qui concerne ces compétences tout au long de l'année. Alors, formateurs, étudiants, futurs-enseignants et enseignants peuvent utiliser cet outil.

\section{Description de la grille :}

Notre grille comporte 30 items qui permettent d'évaluer les étudiants du diplôme général en prenant des notes pendant le cours de méthodologie en langue française. (cf n.1) . L'observateur doit mettre une note dans la case correspondant au niveau atteint par l'étudiant pour chaque item : on a laissé quelques lignes pour la notation des commentaires à la fin de la grille. 


\section{Validité et fidélité de la grille :}

Quant à la validité de la grille élaborée : cet outil a été valide par 4 formateurs. Leurs recommandations et les données recueillies ont permis l'apparition de la grille dans sa forme finale claire.

Pour la fidélité de la grille, la chercheuse a calculé le coefficient de concordance entre les scores attribués par deux observateurs pendant deux observations qui se font simultanément sur un échantillon de 10 étudiants, d'après l'équation suivante (El-Bahey, 1979) : Le coefficient de concordance $=\mathrm{NA} / \mathrm{NA}+\mathrm{ND} \times 100$

Le coefficient de concordance est $80 \%$, ce qui montre que la grille est assez fidèle.

\section{Résultats de l'observation :}

Les résultats de l'observation montrent qu'il y a des difficultés communes chez les étudiants membres de l'échantillon en ce qui concerne les compétences de la prise de notes :

- Difficulté de décodage : $80 \%$ étudiants ont peur de ne pas suivre le cours et d'écouter l'enseignant. Cela dépend du degré de concentration de chaque étudiant.

- Difficultés relatives au sens : dues au lexique. 90\% étudiants possèdent un vocabulaire pauvre, ils ne peuvent pas saisir le sens de la phrase ou du mot.

- Difficultés relatives à la reformulation : 80\% n'arrivent pas à interpréter un mot pour rédiger l'idée de l'enseignant, ils sont obligés à écrire mot à mot et par conséquent ils perdent le fil et le sens. 
- Difficultés de transformation des textes en schéma ou tableau : 70\% ne peuvent pas identifier le thème principal et les idées secondaires qui composent ce thème d'une manière organisée en schéma ou tableau.

\section{2) Elaboration d'un pré/post test :}

La chercheuse a élaboré un pré/post test qui a été appliqué auprès d'un échantillon de 10 étudiants du diplôme général à la faculté de pédagogie université Ain-Chams.(cf.n.2)

\section{Objectifs du test :}

- L'objectif de notre pré/post test est de détecter le niveau actuel des compétences de la prise de notes chez les étudiants du diplôme général à la faculté de pédagogie université Ain-Chams.

- L'objectif de sa ré application est d'évaluer l'efficacité du module basé sur l'APC pour développer les compétences en question.

\section{Description du test :}

Le pré/post test a été élaboré à partir des éléments dégagés de notre problématique ainsi que notre cadre théorique. Il est compose de dix paragraphes dont les étudiants doivent faire la transcription de ce qu'ils lisent et entendent, donc ils doivent suivre la nature des compétences visées.

\section{Passation du test:}

La chercheuse a choisi les étudiants du diplôme général du département de français à la faculté de pédagogie à l'université Ain- 
Chams pendant l'année universitaire 2018-2018 comme échantillon de la recherche actuelle. (Ils sont au nombre de 10)

\section{Durée du test:}

Pour déterminer la durée de l'application du test, la chercheuse a calculé la moyenne du temps pris par le premier et le dernier étudiant pour répondre à toutes les questions. Elle a trouvé que le temps consacré à l'application du test était 40 minutes.

\section{Fidélité du test :}

- Pour calculer l'indice de fidélité du test des compétences de la prise de notes, nous avons fait deux passations à la fin de Février 2018, deux semaines après, nous avons calculé la corrélation entre les deux passations en suivant la formule statistique de Pearson (EI Bahy, 1979).

$$
\mathrm{R}=\quad \frac{\mathrm{NT}-(\mathrm{x})-(\mathrm{y})}{\left.\sqrt{\left(\mathrm{Nx}^{2}-(\mathrm{x})^{2}\right.}\right]\left[\mathrm{Ny}^{2}-(\mathrm{y})\right.}
$$

Donc, la Fidélité était de $85 \%$. Cela signifie que le test est assez

fidèle.

$\underline{\text { Validité du test }}$ : Nous avons calculé la Validité du test à partir de sa Fidélité en appliquant la formule suivante (EI Bahy, 1979).

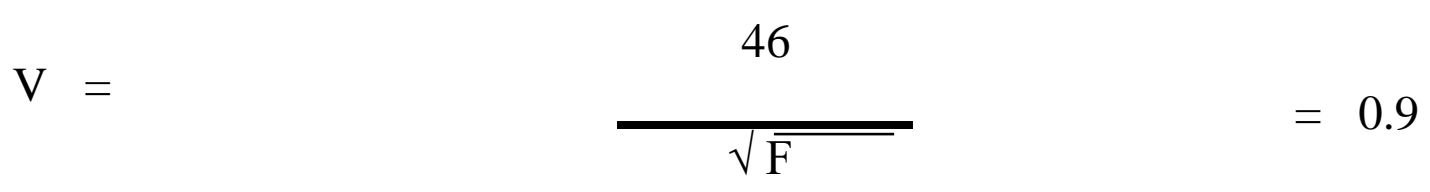

Cette valeur confirme que notre test est valide.

\section{3) Le module proposé :}


Le module pédagogique est une des modalités de l'APC : il est considéré comme la modalité la plus efficace étant vu comme unité didactique indépendante et suffisante, il correspond mieux aux principes de l'autonomie, il se concentre sur l'étudiant, et il n'exige pas de temps supplémentaire.( Richard,M. et Bissonnette,S.2001)

\section{a) Objectifs :}

\section{- Objectif général :}

Le module proposé basé sur l'APC vise à développer les compétences de la prise de notes. Il permet aux étudiants du diplôme général d'acquérir les compétences visées dont ils ont besoin pour intégrer de façon optimale les différentes composantes de l'université.

\section{- Objectifs opérationnels :}

Ce module vise à aider ces étudiants à :

- Savoir prendre des notes dans diverses circonstances : cours, texte, appel téléphonique....

- Savoir retranscrire ses notes (reformuler).

- Faciliter l'enregistrement des informations.

- Faciliter la mémorisation des données essentielles.

- Relever le plus rapidement possible les idées essentielles du cours.

- Encourager l'écoute active pendant l'exposé oral.

- Créer un message à partir d'une prise de notes.

\section{b) Contenu du module :}


مجلة دراسات في المناهج وطرق التّريس، الترقيم الدولي (ISSN 2535-213X)/ العدد 245 (2019))

La chercheuse a choisi le contenu du module parmi les sujets des cours de méthodologie enseignés aux étudiants du diplôme général :

- La gestion de classe.

- Les stratégies d'apprentissage.

- Les stratégies d'enseignement.

- La communication verbale et non verbale.

- L'approche différenciée.

c) Stratégie d'enseignement du module à la lueur de l'APC:

L'enseignement de notre module basé sur l'APC passe par quatre étapes:

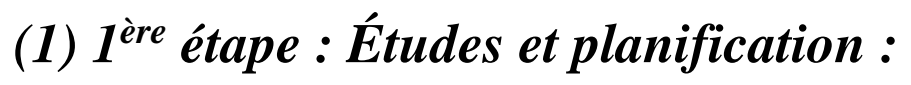

Objectif : elle permet de recueillir l'ensemble des données sur le contexte étudié de formation visée et de déterminer les besoins de formation du public visé.

\section{Rôle de l'étudiant :}

- Choisir son matériel.

- Choisir la technique adaptée à la situation.

- Utiliser des tableaux préparatoires.

- Etre attentif à l'enseignant.

- Respecter le plan du cours.

- Relire les notes du cours précédent. 
مجلة دراسات في المناهج وطرق التّريس، الترقيم الدولي (ISSN 2535-213X)/ العدد 245 (2019))

\section{Rôle de l'enseignant:}

- Remobiliser et réactiver les informations antérieures des étudiants.

- Distribuer une feuille qui contient les abréviations et les symboles les plus fréquents.

- Bien planifier son cours.

- Rappeler aux étudiants qu'ils doivent se servir de leurs habiletés en écoute active si les notes portent sur un document écrit.

\section{(2) $2^{\text {ème }}$ étape : Conception et production du module proposé:}

Objectif: Qui s'appuie sur l'analyse de situation d'apprentissage pour déterminer les compétences que doivent développer l'enseignant chez le public visé et élaborer le module proposé conséquent.

\section{Rôle de l'étudiant:}

- Résumer sans interpréter.

- Restituer ses notes.

- Utiliser les différentes techniques : style télégraphique, carte heuristique, abréviations, symboles....etc.

- Ressortir les éléments essentiels : date, termes nouveaux.

- Saisir les mots clés.

- Sélectionner et créer des reformulations personnelles.

\section{Rôle de l'enseignant:}


مجلة دراسات في المناهج وطرق التثريس، الترقيم الدولي (ISSN 2535-213X)/ العدد 245 (2019)

- Présenter aux étudiants un texte pris en notes.

- Proposer aux étudiants de courts extraits de textes et leur demander de les transcrire sous formes de notes.

- Bien présenter son cours.

- Inciter sur les idées majeures en utilisant : Attention. Il est important de noter que....

\section{(3) $3^{\text {ème }}$ étape : Implantation du module proposé :}

Objectif : Étape où les conditions de réussite doivent être réunies pour la mise en œuvre du module proposé.

\section{Rôle de l'étudiant :}

- Revoir le cours la veille.

- Poser des questions et répondre aux questions.

- Déterminer l'abréviation qui convient le mieux.

- Utiliser différents signes personnels.

\section{Rôle de l'enseignant :}

- Ne pas aller trop vite.

- Encourager et motiver les étudiants.

- Leur fournir les différentes techniques.

- Mettre l'accent sur l'important tout au long du cours. 
مجلة دراسات في المناهج وطرق التّريس، الترقيم الدولي (ISSN 2535-213X)/ العدد 245 (2019))

\section{(4) $4^{\text {ème }}$ étape : Évaluation du module proposé :}

Objectif: elle permet d'évaluer la pertinent, la cohérence et l'efficacité de notre module et de procéder à son réajustement ou à sa mise à jour si besoin.

\section{Rôle de l'étudiant :}

- Corriger les fautes d'orthographe.

- Améliorer le schéma ou le croquis.

- Compléter le cours selon ses besoins.

- Apprendre les termes nouveaux.

- Reformuler les phrases télégraphiques pour apprendre.

- Donner-leur l'occasion de paraphraser et de résumer oralement avant de mettre l'information par écrit.

- S'entrainer aux compétences de la prise de notes à la maison.

\section{Rôle de l'enseignant:}

- Distribuer un corrigé aux étudiants comme évaluation.

- Comparer les différents moyens utilisés par les étudiants pour favoriser la transcription.

- Reformuler le passage difficile par les différentes techniques.

- Se servir de leurs notes et de leurs organisateurs graphiques pour se préparer aux tests.

\section{Application du post/test :}


À la fin de l'application de notre module proposé, nous avons ré appliqué le même test aux membres de notre échantillon afin de vérifier l'efficacité de l'APC sur le développement des compétences de la prise de notes.

\section{Les résultats et l'analyse statistique :}

Après l'application du module proposé, la chercheuse a réappliqué le pré/post test de compétences de la prise de notes sur l'échantillon visé en vue de vérifier son efficacité du module. On a procédé à une analyse statistique des scores des étudiants au pré/post test.

\begin{tabular}{|l|l|}
\hline $\mathbf{R}$ & Rangs \\
\hline $\mathbf{N}$ & Nombre \\
\hline MR & Moyen Rangs \\
\hline $\mathbf{W}$ & Somme des Rangs \\
\hline $\mathbf{E}$ & Écart type \\
\hline $\mathbf{Z}$ & Valeur de "Z" \\
\hline S & Signification \\
\hline $\mathbf{R}$ & Taille de l'effet \\
\hline
\end{tabular}

Tableau № (1)

\begin{tabular}{|c|c|c|c|c|c|c|c|c|c|c|}
\hline \multirow{2}{*}{$\mathbf{R}$} & \multirow{2}{*}{$\mathbf{N}$} & \multirow{2}{*}{ M R } & \multirow{2}{*}{ W } & \multicolumn{2}{|c|}{$\mathbf{M}$} & \multicolumn{2}{|c|}{ É } & \multirow[t]{2}{*}{$\mathbf{Z}$} & \multirow[t]{2}{*}{$\mathbf{S}$} & \multirow[t]{2}{*}{$\mathbf{R}$} \\
\hline & & & & Pré & Post & Pré & Post & & & \\
\hline $\mathbf{R}+$ & 10 & 5.00 & 55 & \multirow{4}{*}{2.40} & \multirow{4}{*}{15.30} & \multirow{4}{*}{2.11} & \multirow{4}{*}{1.42} & \multirow{4}{*}{$\begin{array}{c}2.82 \\
5\end{array}$} & \multirow{4}{*}{0.005} & \multirow{4}{*}{$\begin{array}{c}1.00 \\
\text { saillan } \\
\text { te }\end{array}$} \\
\hline R- & 0 & 0.00 & 0.00 & & & & & & & \\
\hline Neutre & 0 & 0.00 & 0.00 & & & & & & & \\
\hline Total & 10 & & & & & & & & & \\
\hline
\end{tabular}

En utilisant le test de Wilcoxon, le tableau ci-dessus montre que le niveau significatif est plus petit que (0.01). Ce qui signifie qu'il existe une différence statistiquement significative entre les moyennes des notes de l'échantillon de la recherche en ce qui concerne les compétences de la 
prise de notes dans le pré/ post test en faveur du post-test. Pour vérifier l'efficacité de la stratégie proposée pour développer les compétences visées chez l'échantillon de la recherche. De même pour trouver la puissance de la corrélation entre les deux variables indépendante et dépendante, on utilise la corrélation Rank-Biserial qui peut être calculée à travers la formule suivante (Ezzat abdel Hamid, 2011 :279-288) :

$$
r=(4(T 1) / n(n+1))-1
$$

(r)

\begin{tabular}{|l|l|}
\hline $\mathrm{R}$ & Taille de l'effet \\
\hline T1 & Somme des rangs positifs \\
\hline $\mathrm{N}$ & Nombre de paires des donnés de l'échantillon \\
\hline
\end{tabular}
0.4 l'effet est faible.

$0.4 \leq(\mathrm{r})<0.7$ l'effet est modéré.

$0.7 \leq(\mathrm{r})<0.9 \quad$ l'effet est saillant.

$(\mathrm{r}) \geq 0.9 \quad$ l'effet est très saillant.

Après avoir calculé la taille de l'effet, on a trouvé qu'il est égal à (1.00). Ce qui signifie que la stratégie proposée a un effet très saillant sur le développement des compétences de la prise de notes chez les membres de notre échantillon.

Bien que la taille de l'effet soit très forte, ce qui signifie que la stratégie proposée a un effet très fort sur le développement des compétences de la prise de notes, la chercheuse a eu recours à l'équation "Modified Black’s Gain Ratio" (Ezzat abdel Hamid, 2016 :279) :

$M G=\frac{M 2-M 1}{\mathrm{P}-\mathrm{M} 1}+\frac{M 2-M 1}{P}$

$\mathrm{MG}=$ taux de gain modifié

M1 = moyens des notes des participants à la pré-application du test 
M2 = moyens des notes des participants à la post-application du test $\mathrm{P}=$ note totale $\mathrm{du}$ test

Tableau № (2)

\begin{tabular}{|l|l|c|r|c|c|}
\hline \multirow{2}{*}{ La compétence } & $\begin{array}{l}\text { note } \\
\text { totale }\end{array}$ & \multicolumn{2}{|c|}{ moyens des notes } & $\begin{array}{c}\text { taux de } \\
\text { gain } \\
\text { modifié }\end{array}$ & $\begin{array}{c}\text { Significatio } \\
\text { n }\end{array}$ \\
\hline la prise de notes & $\mathbf{2 0}$ & $\mathbf{2 . 4 0}$ & $\mathbf{1 5 . 3 0}$ & 1.37 & Signifiant \\
\hline
\end{tabular}

Nous pouvons constater à partir de ce tableau que le taux de gain modifié de Black de la prise de notes est plus grand que (1.2). Ce qui signifie que la stratégie proposée a un effet très fort sur le développement des compétences de la prise de notes chez les étudiants du diplôme général du département du français à la faculté de pédagogie université d'Ain-Chams.

\section{Interprétation des résultats :}

À travers l'analyse détaillée des résultats, on peut conclure que notre module proposé basé sur l'approche par compétence avait une efficacité positive considérable sur le développement des compétences désignées chez les membres de l'échantillon de la recherche.

En fait, la majorité des participants ont trouvé que le contenu répond tout à fait à leurs attentes et les aide à mieux accomplir et réinvestir leur travail. Ils estiment que l'APC leur a permis d'acquérir des compétences de la prise de notes. D'autres déclarent que l'APC est une nouvelle et différente méthode d'apprentissage et affirment que les compétences développées dans les séances d'APC sont applicables.

Autre aspect positif, le fait que les membres de l'échantillon remplissent un bilan après chaque séance d'APC constitue un bénéfice 
non négligeable dans le développement des compétences visées chez les membres de l'échantillon.

De plus, après l'expérimentation, l'enseignant a un rôle différent : il encourage et motive ses étudiants, leur fournit des différentes techniques, remobilise et réactive leurs informations antérieures. L'étudiant, de sa part, choisit son matériel, restitue ses notes, ressortit ce qui est essentiel, sélectionne des reformulations personnelles, paraphraser et résumer. On peut conclure que le contenu, les méthodes, les procédures utilisées pour la mise en pratique, les méthodes d'évaluation des objectifs ont contribuées a l'acquisition et à l'intégration de nouvelles connaissances, ainsi qu'au développement des compétences de prise de notes.

\section{Conclusion :}

Il vaut toujours mieux prendre notes aux cours. La prise de notes aide les étudiants à garder l'attention pendant le cours et facilite l'assimilation de la matière. Il est capital de maîtriser les notions des cours précédents pour comprendre la nouvelle matière. Avant le cours: un rapide survol de la matière qui doit être traitée permet d'en avoir une idée générale. Après le cours: relire les notes le plus rapide possible pour vérifier si on a tout compris.

Les conclusions de cette recherche mettent en évidence l'effet non négligeable et positif de la prise de notes en contexte d'apprentissage, dans la mesure où il est éprouvé que les étudiants qui prennent de notes lors d'un cours font de meilleurs taux de rappel que les étudiants qui n'en prennent pas. Par conséquent, la prise de notes joue un rôle primordial dans l'acquisition du savoir puisque c'est une activité qui favorise la mémorisation. 
De plus, la prise de notes est un aide-mémoire interne qui s'explique selon les chercheurs par trois causes: la charge cognitive et attentionnelle que cette activité scripturale requiert, la sélection des informations pertinentes à sélectionner, l'organisation de la reformulation effectuée par le noteur durant l'activité.

L'objectif essentiel du module didactique proposé : écrire vite et juste. On entraine les étudiants à recourir à une liste d'abréviations et des signes conventionnels, à utiliser la carte heuristique et le style télégraphique. Chaque étudiant doit construire son code qui lui permet de prendre des notes en accord avec son mode d'intégration des explications données en cours. Mais, on doit faire attention à ne pas transformer la prise de notes en une activité mécanique à réaliser le plus vite possible sans rien comprendre. Le but n'est pas du tout écrire mais d'écrire du sens.

Notre travail permet d'orienter les recherches sur les préconisations à mettre en place pour faciliter la transmission et la mémorisation des connaissances. À côté des pistes de recherche que nous venons d'évoquer , nous souhaitons proposer quelques suggestions pour la formation inspirées des travaux de Mangiante,J. et Parpette,C.(2011) sur le FOU. Ces travaux visent à favoriser la compréhension de la prise de notes des étudiants. Parallèlement, cela permet d'indiquer aux enseignants quels sont les types d'exemples qui sont privilégiés dans la prise de notes par les étudiants afin de pouvoir appréhender et guider l'enseignant en amont dans l'élaboration de son cours.

\section{Bibliographie :}

Albuquerque,C. (2015).De cours de français sur objectifs spécifiques en milieu universitaires. 
Alfarwy,L.(2017) .La didactique du Fos en Syrie : enjeux et perspectives.

Beacco, J-C.( 2007). L'approche par compétences dans l'enseignement des langues. Paris : Didier.

Bertocchini, P., \& Costanzo, E. (2008). Manuel de formation pratique pour le professeur de FLE : cours activités corrigés. Paris : CLE International.

Besancon,M.(2004).Interaction en éducation physique et sportive : approche par competences et construction des savoirs.

Bessonnat, D. (1995). La prise de notes au collège. Pratiques, 86, 53-70.

Boch, F. (2000). Prise de notes et écriture conceptuelle à l'université. Pratiques, 105/10.

Boch,F.(2018) .Pratiques d'écriture et de réécriture à l'université : la prise de notes, entre texte source et texte cible.

.. (2000) . Prise de notes et écriture conceptuelle à l'Université.

Pratiques

Borde,W.(2016).Le dispositif intégré DNL en français /cours de FOS et de FOU dans les FUF bi-plurilingues pour la réussite des étudiants allophones.

Borde, W., Goes, J., \& Mangiante, J.-M. (2016). Le français sur objectif universitaire : entre apports théoriques et pratiques de terrain. Arras : Artois Presses Université.

Bouchet,k.(2018) .Repenser la prise de notes pour les étudiants internationaux en contexte académique : implémentation du dispositif collaboratif Unipad à l'IEP de lyon.

explication Et D

Appliquée, 83, 1991. 
Bouchard, R. et Parpette, C.(2007) « Gestion lexicale et prise de notes dans les cours magistraux : le cas des CM de droit », LIDIL n ${ }^{\circ} 35$.

Bouhadiba F. 2004. Cahiers de langue et de littérature, numéro spécial. Université de Mostaganem.

Boutin, G. \& Julien L. (2000). L'obsession des compétences. Montréal : Éditions Nouvelles.

Bronckart, Jean-Pierre.(2011). Activité langagière, textes et discours. Pour un interactionisme.

Bruno,G.(2012).Impact de l'approche par compétences sur le développement socio économique environnemental : quelles postures pour l'enseignant.

Buffault, B. et alii, (2011). « Pédagogie par objectifs ». [en ligne].

Disponible sur http://cueep. univ-lille1.fr/pédagogie/La_PPO.htm [Consulté le 9 janvier 2014]. Cadre européen commun de référence pour les langues. 2001. Paris : Didier

CUQ J-P., GRUCA I(2005). Cours de didactique du français langue étrangère et seconde. Grenoble : PUG.

sociodiscursif, Paris : Delachaux et Niestlé, 1997, p. 137-138.Cuq, JeanPierre. 2003.Dictionnaire de didactique du français langue étrangère et seconde, CLE international.

Cuq, Jean-Pierre.(2003). Dictionnaire de didactique du français langue étrangère et seconde, CLE internationnal.

seconde, Collection FLE, Grenoble : PUG, 2003, p.165.

l'analyse des besoins à l'élaboration d'un cours, Paris : Hachette FLE, Collection F.Français sur Objectifs Universit Le français sur objectif universitaire Lamia BOUKHANNOUCHE Université Saad-Da Dascalu, E. (2008). Colloque ACEDLE, Association des Chercheurs et Enseignants Didacticiens des Langues Etrangères, Recherches en 
didactique des langues. Université Marc Bloch Strasbourg 2. https://www.

researchgate.net/publication/267790082_Le_francais_sur_objectifs_unive rsitaire.

DE KETELE J-M., et al Guide du formateur, pédagogies en développement. Bruxelles : Editions De Boek, 2007

Delignières, D.(2009). Complexité et compétences. Paris : Editions revue EPS.

Deronne, M. (2012). L'approche par les compétences dans

l'enseignement des mathématiques. Mémoire de master en sciences mathématiques. Université du Mons, France.

Destin,I.(2017).L'approche par compétences en contexte scolaire francophone : Quels enjeux contextuels dans le cas d'Haiti et du Burkina Faso ?

DIONNE, B. (2004)."Prendre des notes en classe", Pour réussir : guide méthodologique pour les études et la recherche, Montréal, Beauchemin, p. 18-29. LB 2369 D592

Dufour, S., \& Parpette, C. (2017). Le cours magistral : interrogations didactiques et analyse de discours. Les Carnets du Cediscor, 13, 61-72. El-Khatib,W.(2009).Mise en place d'une approche par compétences en apprentissage de la langue française dans le contexte libanais : enjeux et difficultés.

ESPINASSE, Marie Chantal. (1998)."La prise de notes; des idées, des conseils, des façons de faire", Parcours sans détour; guide d'accompagnement méthodologique, Montréal, AQPC, p. 151-177. LB 2395 P225 1996

Franic, I., \& Ruet, M. (2014). Le français sur objectif universitaire : du concept à la pratique. Zagreb : FF press. Hafez, S.-A., Chardenet, P., Klett, E., Van Dung, N., \& V. 
Harkat,H. K.(2013) .Le français sur Objectifs Universitaires: quelles sont les stratégies d'apprentissage pour une auto-formation en français chez des étudiants d'économie, en Algérie ?

HILGERT E., 2009. « Un corpus au service du français sur objectifs universitaires : interviews d'enseignants chercheurs», Mélanges CRAPEL, no31, pp. 132-145.

Ivonne ,J.(2018) .Les difficultés des étudiants internationaux dans le système universitaire français. En quoi le Français sur Objectifs Universitaire (FOU) peut-il les aider à réussir leurs études ?

Ghovanlu ,E.F.(2011) .Étude des stratégies linguistiques de locuteurs de français L1 et d'apprenants étrangers lors de la prise de notes en français : perspectives en didactique des langues

Girolami,R.S.(2013).Etude de la prise de notes en anglais par des étudiants francophones : langues étrangères appliquées $2^{\text {eme }}$ année, cours de civilisation américaine.

Gottsmann, L. \& Delignières, D. (2016). A propos des obstacles épistémologiques à l'émergence du concept de compétence. Movement \& Sport Sciences/Science \& Motricité, 94, 71-82. DOI:

$10.1051 / \mathrm{sm} / 2015023$

GUIOMAR, M.G. et D. HÉBERT. (1995)."Prise de notes : document 10", Repères méthodologiques. Aide à l'apprentissage de méthodes de travail, St-Laurent, ERPI, 1995, p. 65 à 68. LB 2395 G964 1995.

Koebel, M. (2006). Réflexions sur quelques enjeux liés à la notion de compétence. Utinam, 6, 53-74.

Le Boterf, G. (1994). De la compétence. Essai sur un attracteur étrange. Paris : Les Editions d'Organisation.

López, J.-S. (2001). Grille d'analyse des manuels / ensembles pédagogiques de FLE. Universidad de Granada (Dpto. de Filología Francesa).

Mangiante, J.-M., \& Parpette, C (2004), Le français sur objectif spécifique, Paris, Hachette. 
...(2007), « Une démarche de

référentialisation en français des professions : le partenariat universités -

Chambre de commerce et d'industrie de Paris (CCIP) »,

www.fdlm.org/fle/ra/articles/modeles.php MANGIANTE J.-M., (2010), Le français dans le monde.

Recherches et applications 47 : Faire des études supérieures en langue française, Paris, CLE International et FIPF. (2011). Le Français sur objectif

universitaire. Grenoble : PUG.

(2012). Le Français sur Objectif

Universitaire : de la maitrise linguistique aux compétences universitaires.

MARTIN R., 2010, «Élaboration et mise en place d'un cours de français sur objectifs spécifiques : FOS - Techniques universitaires », Points Communs, no39,

Mercier, S. (2011). «L'approche par les compétences ».

Moulet,L.(2011).Modélisation de l'apprenant avec une approche par compétences dans le cadre d'environnement d'apprentissage en ligne. MOURLHON-DALLIES, F. (2008), Enseigner une langue à des fins professionnelles, Didier. Le Français sur Objectifs Universitaires - 2011 pp. 135-143 143 Le français sur objectifs universitaires, entre français académique, français de spécialité et français pré-professionnel. . (2008), «Langue de spécialité et logiques professionnelles : enseigner le français en fin de cursus professionnalisant » dans Apprendre une langue de spécialité : enjeux culturels et linguistiques. .. (2006), « Penser le français langue professionnelle », Le français dans le monde, $n^{\circ} 346$ de juillet. FIPF et CLE International, pp. 25-28. 
Notes

\section{Référen}

explication Et Di Nyee-J.Doggen(2018) :L'activité de prise de notes en situation de cours : comparaison des pratiques d'étudiants de Français langue maternelle et de Français langue étrangè Appliquée, 83, 1991. sociodiscursif, Paris : Delachaux et Niestlé, 1997, p. 137Français sur Objectifs U

PARPETTE C., 2004, Le Français sur objectif spécifique : de l'analyse des besoins à l'élaboration d'un cours, Paris : Hachette. Perrenoud, $\mathrm{Ph}$ (2000). L'approche par compétences, une réponse à l'échec scolaire? http://www.unige.ch/fapse/SSE/teachers/perrenoud/php_main/

.................. (2004). L'université entre transmission de savoirs et développement de compétences .Conférence au Congrès de l'enseignement universitaire et de l'innovation, Girona, Espagne, juin 2004.

PIOLAT, Annie.(2001) . La prise de notes. Paris : Presses Universitaires de France.

................ (2004). La prise de notes: Écriture de l'urgence. AixenProvence: Presses Universitaires de Provence. (2006). La prise de notes, 2ième éd., Paris, PUF 128 p. (Coll. que sai-je, 3630) AC 20 Q3 v. 3630

Piolat A., Boch F., (2004) .La prise de notes : Ecriture de l'urgence, in Piolat A., (Dir.), Ecriture Approches en sciences cognitives, Aix-enProvence, Presse Universitaires de Provence, pp.205-229.

Prunet,A.(2018) .Les littéracies en français sur objectifs universitaires : étude d'un corpus contrastif de productions écrites argumentées et perspectives didactiques

Radwan,A.(2017) :La didactique du français sur objectifs universitaires à la croisée des méthodes, entre la conception de manuels et l'élaboration de cours : vers une définition des principes méthodologiques 
Richard M. et Bissonnette S. (2001). Comment construire des compétences en classe. Des outils pour la réforme, (texte condensé du livre). Montréal : Chenelière/McGraw-Hilll.

Roegiers, X. (2000). Une pédagogie de l'intégration: compétences et intégration des acquis dans l'enseignement. Bruxelles: De Boeck Université.

Romainville, M. (1994). Faire apprendre des méthodes : le cas de la prise de notes. Recherche en éducation, 18-19, 37-55. (2000a). L'échec dans l'université de masse. Paris :

L'Harmattan. (2000b). Savoir comment apprendre suffit-il à mieux apprendre?

Said, M.(2015).L'approche par compétences, une approche en apesanteur et/ou les pesanteurs de l'environnement d'implantation ? Le cas du curriculum de français du troisième cycle du système éducatif algérien : pertinence et/ou faisabilité ?

Scallon, G. (1999). L'évaluation formative. Montréal : Éditions du renouveau pédagogique. . (2004). L'évaluation des apprentissages dans une approche par compétences. Saint-Laurent : Éditions du renouveau pédagogique

Slingeneyer, M. (2013). Carnet pratique. Le français dans le mille, 238, 96-99.

TREMBLAY, R et Y. PERRIER.(2000). "Les notes de cours : module 5", Savoir Plus ; Outils et méthodes de travail intellectuel, Montréal, Chenelière / McGraw Hill , p. 37 à 41. LB 2395 T7892

Vlad, M. (2010). Le français sur objectifs universitaires entre globalisation et localisation. In J. Caillier \& S. Borg (Eds.), Forum Mondial Héraclès, Université de Perpignan Via Domitia. Actes du Colloque. Le français sur objectifs universitaires. Synergies Monde, 8(2), (pp. 211-232). 
Suzanne R. Girolami-Galvin(2008) .Etude de la prise de notes en anglais par des étudiants francophones : langues étrangères appliquées 2ème année, cours de civilisation américaine

\section{Sitographie}

http://www.euromonitor.com/travel-and-tourism-in-malawi/report http://www.ambafrance-zm.org/IMG/pdf/FCC_Law_Res-2.pdf http://www.mzuni.ac.mw/index.php?option=com_content\&view=article $\& \mathrm{id}=23 \&$ Itemid $=18$

http://www.etravelworld.cn/malawi/Publications/documents/TOURISM $\%$ 20INVESTMENT\%20OPPORT

UNITIES\%20IN\%20MALAWI.pdf

http://www.zewo.ch/impact/fr/mesure_de_lefficacite/etape1_definir_obje ctifs_projet/Finalite

http://www.cedip.equipement.gouv.fr/definir-des-objectifs-en-formationa21.html

http://www.formaeva.com/files/documents/ressources/definir-objectifs-

formation.pdf

http://mediacla.univfcomte.

fr/GEIDEFile/produit_adnot_final.pdf?Archive=191266891944\&File=pr oduit+Adnot

+final_pdf

http://mediacla.univfcomte.

fr/GEIDEFile/Master2_produit_Mathieu_Baduel.pdf?Archive=19144809 $1962 \&$ File $=$

Master2_produit_Mathieu_Baduel_pdf

http://mediacla.univfcomte.

fr/GEIDEFile/produit_final_k_drapeau.pdf?Archive=191081391926\&Fil e=produit_fi

www.fda.ccip.fr/points-communs/pc-39

http://prezi.com/4m5ofiheqtor/lapproche-par-competence/ 